\section{Acknowledgments}

The authors would like to thank Richard Lifton and Christine Seidman for their advice and contributions to the text.

Address correspondence to: David Altshuler, Department of Molecular Biology, Massachusetts General Hospital, Boston, Massachusetts 02114, USA. Phone: (617) 726-5940; Fax: (617) 726-5937; E-mail: altshuler@molbio.mgh.harvard.edu. Or to: Joel N. Hirschhorn, Enders 561, Children's Hospital, 300 Longwood Avenue, Boston, Massachusetts 02115, USA. Phone: (617) 919-2129; Fax: (617) 730-0253; E-mail: joelh@broad.mit.edu.

1. Lusis, A.J., Mar, R., and Pajukanta, P. 2004. Genetics of atherosclerosis. Annu. Rev. Genomics Hum. Genet. 5:189-218.

2. Wang, L., Fan, C., Topol, S.E., Topol, E.J., and Wang,
Q. 2003. Mutation of MEF2A in an inherited disorder with features of coronary artery disease. Science. 302:1578-1581.

3. Weng, L., et al. 2005. Lack of MEF2A mutations in coronary artery disease. J. Clin. Invest. 115:1016-1020. doi:10.1172/JCI200524186.

4. Bhagavatula, M.R., et al. 2004. Transcription factor MEF2A mutations in patients with coronary artery disease. Hum. Mol. Genet. 13:3181-3188.

5. Wacholder, S., Chanock, S., Garcia-Closas, M., El Ghormli, L., and Rothman, N. 2004. Assessing the probability that a positive report is false: an approach for molecular epidemiology studies. J. Natl. Cancer Inst. 96:434-442.

6. Lohmueller, K., Pearce, C.L., Pike, M., Lander, E.S., and Hirschhorn, J.N. 2003. Meta-analysis of genetic association studies supports a contribution of common variants to susceptibility to common disease. Nat. Genet. 33:177-182.

7. Hirschhorn, J.N., and Altshuler, D. 2002. Once and again-issues surrounding replication in genetic association studies. J. Clin. Endocrinol. Metab. 87:4438-4441.

8. Dahlman, I., et al. 2002. Parameters for reliable results in genetic association studies in common disease. Nat. Genet. 30:149-150.
9. Florez, J.C., Hirschhorn, J., and Altshuler, D. 2003. The inherited basis of diabetes mellitus: implications for the genetic analysis of complex traits. Annu. Rev. Genomics Hum. Genet. 4:257-291.

10. Lifton, R.P., Gharavi, A.G., and Geller, D.S. 2001. Molecular mechanisms of human hypertension. Cell. 104:545-556.

11. Wang, Q., et al. 2004. Premature myocardial infarction novel susceptibility locus on chromosome 1P34-36 identified by genomewide linkage analysis. Am. J. Hum. Genet. 74:262-271.

12. Pajukanta, P., et al. 2000. Two loci on chromosomes 2 and $X$ for premature coronary heart disease identified in early- and late-settlement populations of Finland. Am. J. Hum. Genet. 67:1481-1493.

13. Hauser, E.R., et al. 2004. A genomewide scan for earlyonset coronary artery disease in 438 families: the GENECARD Study. Am. J. Hum. Genet. 75:436-447.

14. Francke, S., et al. 2001. A genome-wide scan for coronary heart disease suggests in Indo-Mauritians a susceptibility locus on chromosome 16p13 and replicates linkage with the metabolic syndrome on 3q27. Hum. Mol. Genet. 10:2751-2765.

15. Broeckel, U., et al. 2002. A comprehensive linkage analysis for myocardial infarction and its related risk factors. Nat. Genet. 30:210-214.

\title{
The fickle finger of fate
}

\author{
Luis de la Fuente and Jill A. Helms
}

Department of Surgery, Stanford University, Stanford, California, USA.

In this issue of the JCI, Niedermaier and colleagues demonstrate that a chromosomal inversion in mice results in dysregulation of Sonic hedgehog (Shh), such that $S h b$ is ectopically expressed in a skeletogenic domain typically occupied by Indian hedgehog (Ihh) (see the related article beginning on page 900). This molecular reversal eliminates phalangeal joint spaces, and consequently, Short digits $(D s h)$ heterozygotes $(D s h /+)$ have brachydactyly (shortened digits). I $b h$ is normally downregulated in regions that will become the joint space, but in $D s h /+$ mice, Shh bypasses this regulatory control and persists; accordingly, cells maintain their chondrogenic fate and the developed digits are shorter than normal. The significance of these data extends far beyond the field of skeletal biology: they hint at the very real possibility that the endogenous $S h b$ regulatory region contains a repressor designed to segregate the activity of Shh from Ihh. The existence of such a repressor provides a window into the distant past, revealing that $S h h$ and Ihh must once have shared responsibilities in establishing tissue boundaries and orchestrating vertebrate tissue morphogenesis.

Ancient physicians viewed the skeleton as "the foundation of the rest of the parts of the body and all the members rest upon them and are supported, as proceeding from a primary base" (1). Defects in this structural foundation also serve as portholes through which the process of

Nonstandard abbreviations used: Bmp, bone morphogenic protein; Dsh, Short digits; Dsh $/+$, Dsh heterozygote; Ihh, Indian hedgehog; Shh, Sonic hedgehog.

Conflict of interest: The authors have declared that no conflict of interest exists.

Citation for this article: J. Clin. Invest. 115:833-836 (2005). doi:10.1172/JCI200524840. fetal skeletogenesis can be analyzed. For example, studies of genetic perturbations that result in distal limb truncations have shown that the morphogen Sonic hedgehog (Shh) establishes precisely where skeletogenic condensations will form in the tips of the hand- and footplates $(2,3)$. This spatial patterning information is further refined by bone morphogenetic protein (Bmp) signaling, as shown by the fact that disruptions in the Bmp signaling pathway lead to fusions, or syndactyly, of the digits (4). Once the spatial pattern of the skeletogenic condensations is achieved, a closely related cousin, Indian hedgehog (Ihh), takes over and plays an instrumental role in segregating inner chondrogenic cells from the flattened, elongated perichondrial cells at the periphery (5). Ihh secreted from chondrocytes stimulates the differentiation of perichondrial cells into osteoblasts (5-7), mesodermal cells that give rise to bone.

Another critical feature of limb skeletogenesis is the creation of the articulations, or joint spaces, between the skeletal elements. In the fingers, joint spaces are created when a single, larger skeletogenic condensation cleaves into 2 or 3 smaller segments, each of which will give rise to a phalange (8). Wnt14 is critical in determining where a joint space will form (9), but precisely how the cleavage event is controlled remains uncertain. One thing is clear, however: when a separation fails to take place, the phenotypic consequence is brachydactyly (shortened digits). Thus, while we have a fairly complete picture of the range of skeletal malformations that can occur, how these disruptions are related to one another and to the basic program of skeletogenesis remains unknown.

\section{Reading the bones}

In this issue of the JCI, Niedermaier et al. provide new insights into the process of 


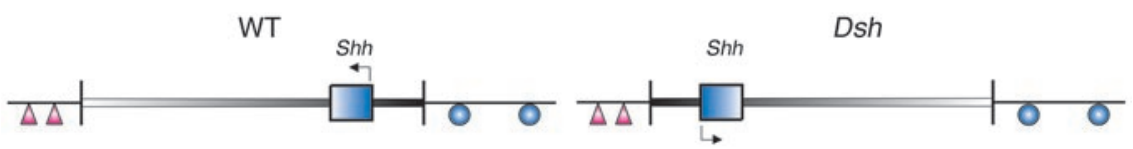

Figure 1

A chromosomal inversion causes Shh relocation. Schematic representation of the inversion event leading to development of Dsh mutant mice; the Shh gene, normally influenced by distal enhancer sequences (blue circles), is moved nearer to other regulatory sequences (pink triangles).

skeletal morphogenesis, describing the phenotypic consequences of a chromosomal inversion that disrupts both temporal and spatial regulation of $S h b(10)$. Chromosomal inversions are genetic mishaps that take place when a region of the chromosome breaks and rejoins in a new orientation (Figure 1). This structural aberration can serve as a window into embryogenesis, especially when genes within the inverted region are subjected to new regulation as a result of enhancers or promoters located on the other side of the breakpoint.

In the current study Niedermaier and colleagues examined heterozygous mice in which an inversion caused foreshortening of the distal limb skeletal elements, hence their nom de plume, Short digits (Dsh) (10). In the homozygous state, the $D s h$ phenotype is far more severe; in fact, $D s h / D$ sh embryos bear a striking resemblance to $S h b^{-/-}$embryos in that both exhibit cyclopia and incomplete cleavage of the embryonic forebrain. But the emphasis in this study was on the skeletal anomalies of the limb; $D s h / D s h$ embryos showed an absence of all distal limb skeletal elements. Because Dsh/+;Shb ${ }^{+/}$ mice had a phenotype nearly identical to that of $S h h^{-/-}$and $D s h / D s h$ mice, the authors concluded that $D s h$ and $S h b$ were allelic.

\section{It's about time; it's about space}

Perhaps the most unexpected finding came from studying the $D s h /+$ phenotype: here, the authors found that the chromosomal

\section{Figure 2}

The chromosomal inversion causes Shh to be expressed ectopically in the phalanges. (A and $\mathbf{B}$ ) In WT embryos (A), Ihh is expressed in mesenchymal aggregates of cells called cartilage condensations, up to the cartilage-perichondrium boundary (dotted lines); whereas in Dsh/+ embryos (B), Ihh is lost from the phalangeal condensations. (C and $\mathbf{D})$ Whereas in WT embryos (C), Shh is never expressed in skeletal condensations, in Dsh/+ embryos (D), Shh expression replaces $\mathrm{Ih} h$, albeit in a larger domain that extends past the cartilage-perichondrium boundary. u, ulna; $r$, radius. inversion functionally removed Shh activity from a number of its endogenous sites of activity, but also directed ectopic Shb expression to - of all places - the region where $I h b$ is normally expressed, the limb skeletogenic condensations (Figure 2) (10). Ectopic expression of Shb occurred simultaneously with the loss of $I b h$ in the digit condensations. While the 2 events - induction of $S h b$ and loss of I $h b-$ might be controlled by separate mechanisms, a more plausible explanation is that the loss of normal $I b h$ expression is a secondary consequence of ectopic Shb expression, since previous investigators have shown that a negative feedback loop controls Hedgehog signaling in the skeleton (11).

Another phenotypic peculiarity was that the molecular substitution of Shb for $I b h$ only took place in the digits, while more proximal skeletal elements (i.e., humerus, radius, and ulna) were spared. Here, the answer may lie in the timing: the limb skeleton develops in a proximal-to-distal direction, and the inversion disrupted Shb expression relatively late in gestation, perhaps after the proximal skeletal elements were specified. But what remains a puzzle - and a very exciting one at that - is just how this inversion resulted in ectopic Shb
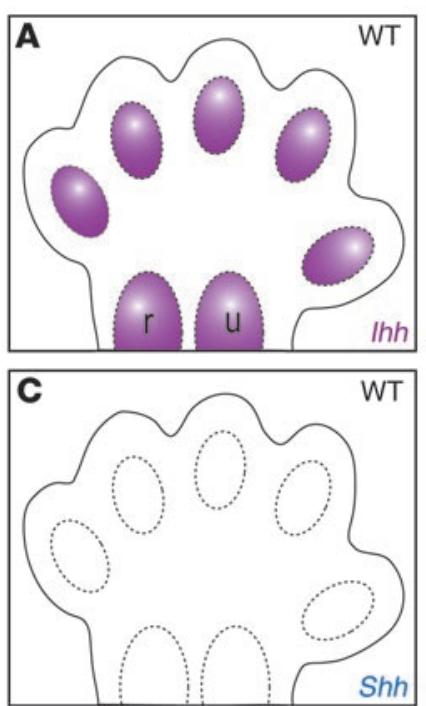

expression in the digits. In other words, where did the instructions driving Shb expression in the digits originate? The chromosomal rearrangement appears to have created a new, chimeric regulatory domain for the Shb gene, composed partially of resident enhancer sequences and partially of enhancer sequences that were translocated along with the $S h b$ gene.

Another perplexing question arises: how was the information in this chimeric regulatory domain derived? One possibility is that the inversion positioned the Shb coding sequence so that it was adjacent to a new enhancer that drives the expression of one or more genes in the digits. This possibility seems unlikely, however, as none of the genes near the insert point were expressed in the digits (10). A more plausible and attention-grabbing possibility is that the instructions for driving spatiotemporal expression of Shh are contained within the normal Shb regulatory region but are somehow masked. If this is the case, then the inversion event may have resulted in the loss of a repressor activity that normally restricts $S h b$ from being expressed in skeletogenic condensations.

The implications of this finding are far reaching: if a repressor sequence was disrupted by chromosomal rearrangement, then the ectopic $S h b$ represents part of a more ancient pattern of expression. Furthermore, these data suggest that the normal Shb regulatory sequence may have buried within it instructions for targeting $S h b$ expression to skeletogenic condensations. That Shb and Ihb might have shared expression patterns sometime in the distant past is not a large stretch, since gene duplication
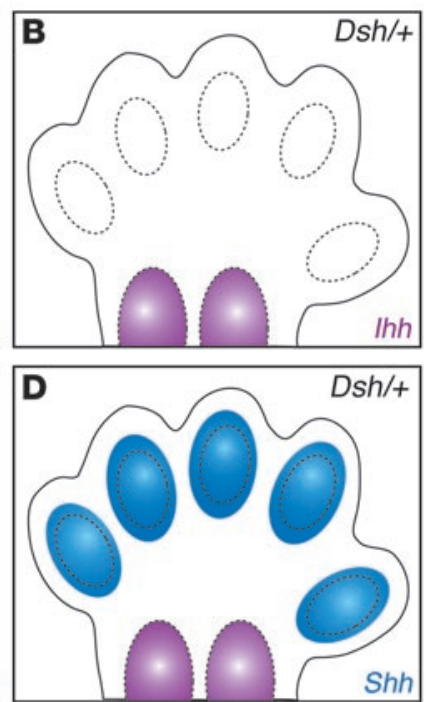


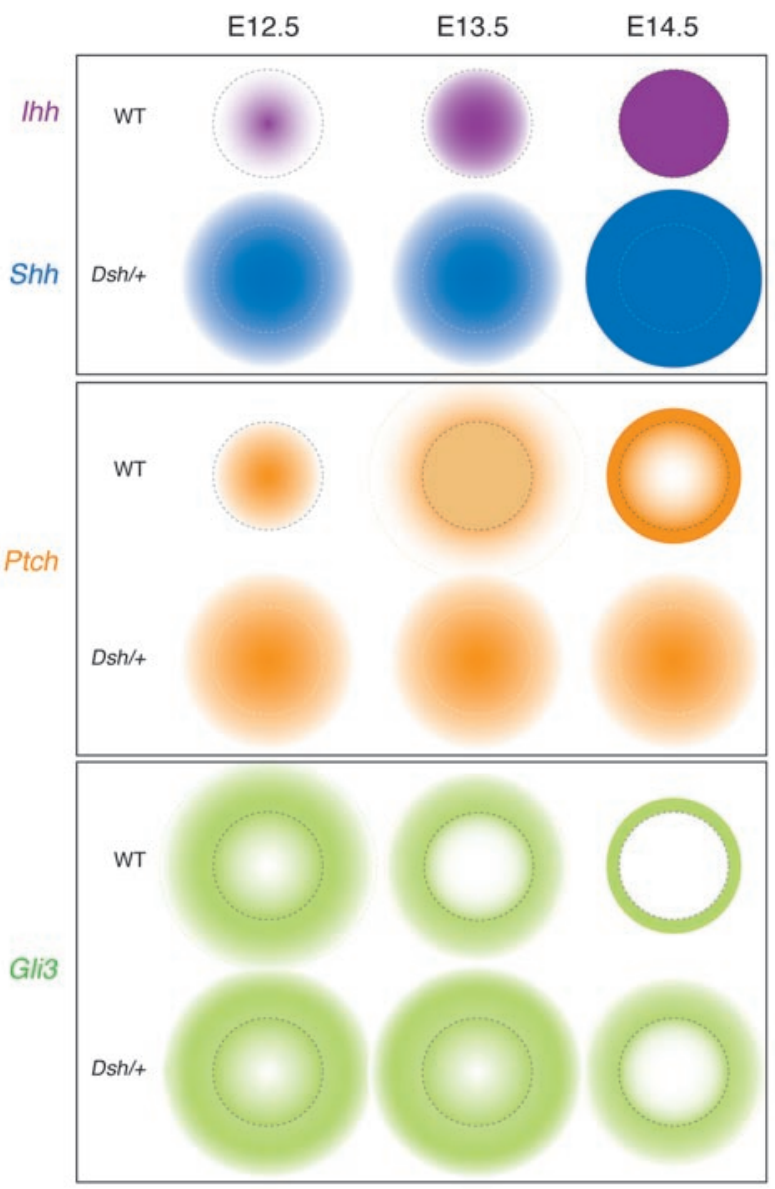

is thought to be one of the chief mechanisms responsible for the diversification of gene function.

\section{Limbs and faces}

The precise regulatory region perturbed by the chromosomal translocation in $D s h /+$ mice is unknown, but it appears to be responsible for controlling ectopic $S h b$ expression in both the limbs and the facial prominences. Niedermaier and colleagues report that $S h b$ is ectopically expressed in the $D s h /+$ frontonasal prominence, which leads to flaws in craniofacial patterning (10). In this anatomical locale, $S h b$ regulates proximodistal outgrowth and mediolateral expansion of the neural crest cell population that resides in the facial midline (12). Eventually these cells will produce the skeletal elements of the middle and upper face, a developmental step that is also dependent upon Hedgehog signaling $(13,14)$. Clinicians and researchers have long been aware of the connection between facial and limb malformations, and a surprisingly large number of syndromes are characterized by anomalies in both craniofacial and

\section{Figure 3}

Disrupted expression boundaries in Dsh/+ animals lead to perichondrial and joint defects (1). A transverse schematic of the phalangeal skeletal condensation, showing expression domains corresponding to a specific Hedgehog gene (Ihh in WT, Shh in Dsh/+), the Hedgehog target Ptch, and the Hedgehog repressor Gli3. Dotted lines indicate the normal cartilage-perichondrium boundary. In WT phalanges, Ihh (purple) is initially expressed (E12.5) in a small domain that expands over time to encompass the skeletal condensation. At E12.5 in Dsh/+ phalanges, Shh expression (blue) encompasses the normal Ihh domain and over time extends beyond what would normally be the cartilage-perichondrium boundary. Ptch (orange), an indicator of Hedgehog responsiveness, is first expressed in WT phalanges coincident with Ihh; eventually, Ptch is downregulated in cells expressing Ihh and upregulated in cells adjacent to the Ihh domain, where it functions to limit the spread of Hedgehog expression. Note the absence of color within the element, which shows the relative lack of Ptch in this domain (10). In Dsh/+ phalanges, Ptch is expressed coincident with the broader domain of Shh; however, in contrast to what occurs in WT tissues, Ptch does not become downregulated in chondrocytes. Gli3 (green), a repressor of Hedgehog signaling, is initially expressed in WT phalanges in a domain overlapping with $/ \mathrm{hh}$; over time, this domain is restricted to the perichondrium, where Gli3 limits the activity of the Hedgehog protein. In $D s h /+$ phalanges the Gli3 expression domain is initially broader, and only after an extended period of time does it become restricted to the perichondrium.

limb structures (15). The Niedermaier study (10) suggests one possible molecular mechanism for this clinical observation: if the same regulatory region controls $\mathrm{Shb}$ expression in the digits and in the face, then both tissues will necessarily be affected by any perturbations, be they genetic or environmental, in that regulatory domain.

\section{A molecular switcheroo}

Niedermaier et al.'s molecular dissection of the $D s h /+$ phenotype has provided additional insights into the molecular underpinning of brachydactyly. Specifically, the investigators show that Shh does not simply replace Ihh in $D s h /+$ chondrocytes; rather, Shh is more widely distributed in the cartilage and perichondrium of the digits, and it is active in this larger domain, as illustrated by the expansion of Hedgehog target genes Gli1 and Ptch (Figure 3) (10).

The consequences of ectopic Hedgehog expression are delayed chondrocyte differentiation, retardation of the maturation of perichondrium into periosteum, and loss of distal joint spaces. Given that all 3 defects occur simultaneously, one cannot help but wonder if they are interrelated features, all initially dependent upon a single precipitating event. We speculate that this event is the establishment of a boundary that segregates chondrocytes and osteoblasts into adjacent but immiscible cell populations. This boundary is, to some extent, a consequence of local Hedgehog signaling.

Around the same time that the cartilageperichondrium boundary is being specified, some cells within the $I h b$-positive skeletal condensation are faced with another decision: whether to progress along a chondrogenic pathway and undergo hypertrophy or to differentiate into a specialized type of perichondrial cell that will line the joint space (16). This cell fate decision, along with formation of a proper perichondrium, is disrupted in the digits of $D s h /+$ mice as a consequence of ectopic Hedgehog activity (10). Just how does this imbalance between Hedgehog responsiveness and repression cause cells to alter their fate? Organisms use boundaries, the juxtaposition of like and unlike cells, in a myriad of ways: to define compartments, convey positional information, and even establish new tissues and signaling centers that can only form at the interface between 2 populations. In a wide variety of other developmental contexts, Hedgehog proteins play a critical role in specifying boundaries and thus defining cell and tissue compartments (17-19); the same may be true during skeletogenesis, but data to support this hypothesis are still sparse. What the Niedermaier et al. study reveals, however, is that removal or expansion of one of the factors that contributes to the establishment of a boundary can cause a multitude of processes, including those that shape and control development of the skeleton, to go awry. 


\section{Acknowledgments}

J.A. Helms is supported in part by the Oak Foundation.

Address correspondence to: Jill A. Helms, Department of Surgery, Stanford University, 257 Campus Drive, GK 207, Stanford, California 94305, USA. Phone: (650) 736-0919; Fax: (650) 736-4374; E-mail: jhelms@stanford.edu.

1. Massa, N. 1536. Liber introductorius anatomiae, sive dissectionis corporis humani. Venetiis. Cap. 21:37-38.

2. Riddle, R.D., Johnson, R.L., Laufer, E., and Tabin, C. 1993. Sonic hedgehog mediates the polarizing activity of the ZPA. Cell. 75:1401-1416.

3. Litingtung, Y., Dahn, R.D., Li, Y., Fallon, J.F., and Chiang, C. 2002. Shh and Gli3 are dispensable for limb skeleton formation but regulate digit number and identity. Nature. 418:979-983.

4. Dahn, R.D., and Fallon, J.F. 2000. Interdigital regulation of digit identity and homeotic transformation by modulated BMP signaling. Science. 289:438-441
5. Colnot, C., et al. 2005. Indian hedgehog synchronizes skeletal angiogenesis and perichondrial maturation with cartilage development. Development. 132:1057-1067.

6. St-Jacques, B., Hammerschmidt, M., and McMahon, A.P. 1999. Indian hedgehog signaling regulates proliferation and differentiation of chondrocytes and is essential for bone formation. Genes Dev. 13:2072-2086.

7. Chung, U.I., Schipani, E., McMahon, A.P., and Kronenberg, H.M. 2001. Indian hedgehog couples chondrogenesis to osteogenesis in endochondral bone development. J. Clin. Invest. 107:295-304.

8. Archer, C.W., Dowthwaite, G.P., and Francis-West, P. 2003. Development of synovial joints. Birth Defects Res. C. Embryo Today. 69:144-155.

9. Hartmann, C., and Tabin, C.J. 2001. Wnt-14 plays a pivotal role in inducing synovial joint formation in the developing appendicular skeleton. Cell. 104:341-351.

10. Niedermaier, M. 2005. An inversion involving the mouse Shh locus results in brachydactyly through dysregulation of Shh expression. J. Clin. Invest. 115:900-909. doi:10.1172/JCI200523675.

11. Vortkamp, A., et al. 1996. Regulation of rate of cartilage differentiation by Indian hedgehog and PTH-related protein. Science. 273:613-622.

12. Hu, D., Marcucio, R.S., and Helms, J.A. 2003. A zone of frontonasal ectoderm regulates patterning and growth in the face. Development. 130:1749-1758.

13. Hu, D., and Helms, J.A. 1999. The role of sonic hedgehog in normal and abnormal craniofacial morphogenesis. Development. 126:4873-4884.

14. Jeong, J., Mao, J., Tenzen, T., Kottmann, A.H., and McMahon, A.P. 2004. Hedgehog signaling in the neural crest cells regulates the patterning and growth of facial primordia. Genes Dev. 18:937-951.

15. Gorlin, R.J., Cohen, M.M., and Levin, L.S. 1990. Syndromes of the head and neck. Oxford University Press. New York, New York, USA. 977 pp.

16. Garciadiego-Cazares, D., Rosales, C., Katoh, M., and Chimal-Monroy, J. 2004. Coordination of chondrocyte differentiation and joint formation by alpha5beta 1 integrin in the developing appendicular skeleton. Development. 131:4735-4742.

17. Dahmann, C., and Basler, K. 2000. Opposing transcriptional outputs of Hedgehog signaling and engrailed control compartmental cell sorting at the Drosophila A/P boundary. Cell. 100:411-422.

18. Sarkar, L., et al. 2000. Wnt/Shh interactions regulate ectodermal boundary formation during mammalian tooth development. Proc. Natl. Acad. Sci. U. S. A. 97:4520-4524.

19. Larsen, C.W., Hirst, E., Alexandre, C., and Vincent, J.P. 2003. Segment boundary formation in Drosophila embryos. Development. 130:5625-5635.

\section{Altered regulation of IL-2 production in systemic lupus erythematosus: an evolving paradigm}

Gary M. Kammer

Arthritis Associates Inc., Willoughby, Ohio, USA.

In systemic lupus erythematosus (SLE), IL-2 production by T lymphocytes in vitro is impaired. Deficient IL-2 production may be an outcome of a primary SLE T cell disorder that is due to impaired signal transduction. In this issue of the JCI, evidence is presented that an anti-TCR/CD3 complex autoantibody present in SLE sera can bind to $T$ cells and activate the $\mathrm{Ca}^{2+}$-calmodulin kinase IV (CaMKIV) signaling cascade, resulting in downregulation of $I L-2$ transcription and IL-2 production (see the related article beginning on page 996). Because IL-2 may contribute to the maintenance of T cell tolerance, deficient IL-2 production could promote a breach of $\mathrm{T}$ cell tolerance that results in autoantibody production in SLE.

Recently, it has been recognized that diverse autoantibodies directed against intra- and extracellular autoantigens exist in patients with systemic lupus erythematosus (SLE) for years before the clinical diagnosis is made (1); this suggests that physiologic mechanisms

Nonstandard abbreviations used: CaMKIV, $\mathrm{Ca}^{2+}$ calmodulin kinase IV; CRE, cAMP response element; CREB, CRE-binding protein; CREM, CRE modulator; IL-2R, IL-2 receptor; PCREB, phosphorylated CREB; pCREM, phosphorylated CREM; PKA-II $\beta$, type II $\beta$ PKA; pRII $\beta$, phosphorylated RII $\beta$; RII $\beta$, $\beta$ type II regulatory subunit; SLE, systemic lupus erythematosus.

Conflict of interest: The author has declared that no conflict of interest exists.

Citation for this article: J. Clin. Invest. 115:836-840 (2005). doi:10.1172/JCI200524791. that maintain tolerance to self antigens have been breached. Tolerance to self antigens is established and preserved by a subpopulation of T lymphocytes known as Tregs (2), and the loss of tolerance is a pathologic process giving rise to autoimmunity. This circumstance raises the possibility of the existence of abnormal $\mathrm{T}$ cell clones that mediate defective helper and suppressor effector functions, which result in autoantibody generation by forbidden $\mathrm{B}$ cell clones. In SLE, defective signaling cascades are believed to give rise to a primary $\mathrm{T}$ cell disorder that is characterized by impaired effector functions (3). These effector dysfunctions are, at least in part, a result of skewed expression of various effector molecules, including CD40 ligand (e.g., CD154) and multiple cytokines, and may reflect an imbalance of gene expression. An extracellular factor(s) in the microenvironment that interacts with $\mathrm{T}$ cells and exacerbates these dysfunctions has not been previously identified.

\section{Tregs, skewed cytokine production, and loss of tolerance}

Impaired effector $\mathrm{T}$ cell functions due to skewed cytokine production may create a microenvironment that promotes a strong Th2 immune response relative to Th1 and Treg activity. Relative overproduction of IL-4, IL- 6 , and IL- 10 by Th 2 cells and underproduction of IL-2, IL-12, TGF- $\beta$, and IFN- $\gamma$ by Th1 cells and Tregs can result in imbalanced autocrine and paracrine effects on $\mathrm{T}$ and $\mathrm{B}$ cells in the microenvironment. Because of the reduced numbers of $\mathrm{CD} 4{ }^{+} \mathrm{CD} 25^{+}$Tregs (4) as well as the diminished generation of IL-2 and TGF- $\beta$, there may be insufficient suppressor activity in SLE to counterbalance the enhanced Th2 effect on B cell antibody production. Taken together, these conditions create a microenvironment that promotes a 